\title{
A New Spin on Red Giant Rapid Rotators: Evidence for Chemical Exchange Between Planets and Evolved Stars
}

\author{
Joleen K. Carlberg ${ }^{1}$, Steven R. Majewski ${ }^{1}$, Verne V. Smith ${ }^{2}$, \\ Katia Cunha ${ }^{2}$, Richard J. Patterson ${ }^{1}$, Dmitry Bizyaev ${ }^{3}$, Phil Arras ${ }^{1}$, \\ and Robert T. Rood ${ }^{1}$ \\ ${ }^{1}$ Dept. of Astronomy, University of Virginia, Charlottesville, VA 22904 \\ ${ }^{2}$ National Optical Astronomy Observatory, Tucson, AZ, 85719 \\ ${ }^{3}$ Apache Point Observatory, Sunspot, NM, 88349
}

\begin{abstract}
Rapid rotation in red giant stars may be one signature of the past engulfment of a planetary companion. Models of the future tidal interaction of known exoplanet host stars with their planets show that many of these stars will accrete one or more of their planets, and the orbital angular momentum of these accreted planets is sometimes sufficient to spin up the host stars to a level commonly accepted as "rapid rotation" for giant stars. Planets accreted during the red giant phase should leave behind a chemical signature in the form of unusual abundance patterns in the host red giant's atmosphere. Proposed signatures of planet accretion include the enhancement of $\mathrm{Li}$ and ${ }^{12} \mathrm{C}$; both species are generally depleted in giant star atmospheres by convection but could be replenished by planet accretion. Moreover, accreted planets may preferentially enhance the stellar abundance of refractory elements assuming that the refractory nature of these elements leads to their relative enhancements in the planets themselves. Here we present preliminary results of a search for these predicted chemical signatures through high resolution spectroscopic abundance analysis of both rapidly rotating giant stars (i.e., stars with a higher probability of having experienced planet accretion) and normally rotating giant stars. We find that the rapid rotators are enhanced in Li relative to the slow rotators - a result consistent with Li replenishment through planet absorption.
\end{abstract}

Keywords. stars: abundances, stars: chemically peculiar,stars: rotation

\section{Background}

Generally, red giants are slow rotators with $v \sin i \approx 2 \mathrm{~km} \mathrm{~s}^{-1}$. A small fraction of red giants, however, have $v \sin i$ in excess of $8 \mathrm{~km} \mathrm{~s}^{-1}$; these are the "rapid rotators." Planet accretion is a possible mechanism for spinning up rapidly rotating giants. Modeling the tidal interaction of exoplanet host stars and their planets, Carlberg et al. (2009), hereafter C09, found that the lower red giant branch (RGB) was one of the best late stellar evolutionary stages to find evidence for planet induced rapid rotation. Giants are good candidates for searching for chemical evidence of this accretion because they are chemically distinct from planets in ways that main sequence stars are not. Giant stars process light elements, reducing their surface abundances up to several orders of magnitude. Consequently, a planet with unprocessed abundances of these light elements can significantly enhance a giant star's atmospheric abundance. For this work, we focus on lithium, an element that is easily measured in the optical spectra of giant stars. 


\section{Observations and Analysis}

To test the idea that the rapid rotation seen in some field red giant stars is caused by past planet accretion, we conducted a systematic survey of both rapid rotators and normal slow rotators to look for global differences between these two samples. New rapid rotator candidates were identified by cross-correlating spectra of $\sim 1300$ red giants against an Arcturus template and analyzing the width of the cross-correlation peak (Carlberg, et al. 2009, in prep.). These giants were originally observed by Bizyaev, et al. (2006) at high-resolution but low signal-to-noise $(S / N \sim 20-40)$. Additional stars were selected from de Medeiros \& Mayor (1999) to bolster the sample size with some brighter targets. The control sample of slow rotators was built from the same sources. Over 100 of these slow and rapid rotators were then reobserved at $S / N \geqslant 100$ at the Kitt Peak 4-m and APO 3.5-m telescopes with echelle spectrographs of $R \sim 43,000$ and 31,500, respectively.

Using Kurucz (1994) model atmospheres and the Sneden (1973) stellar line analysis program, MOOG, we derived the stellar parameters of our stars using $74 \mathrm{Fe} \mathrm{I}$ and 13 Fe II lines. The stellar parameters $\left(T_{e f f}, \log g,[\mathrm{Fe} / \mathrm{H}]\right.$, and $\left.\xi\right)$ were given by the stellar atmosphere model for which the neutral iron abundance, A(Fe I), was independent of iron lines' excitation potentials and reduced equivalent widths and for which the derived $\mathrm{A}(\mathrm{Fe} \mathrm{I})$ and $\mathrm{A}(\mathrm{Fe} \mathrm{II})$ matched the input metallicity of the model. The rotational and macroturbulent velocities were derived from the iron line at $6750.15 \AA$, an unblended line in the same echelle order as lithium. Spectral synthesis was used to derive the lithium abundance from the resonance lines at $6707 / 8 \AA$. We have derived stellar parameters and lithium abundances in a subsample of 26 of our stars: 9 rapid rotators and 17 slow rotators, which were all chosen without previous knowledge of the stellar lithium content.

\section{Results and Discussion}

In Figure 1 we plot the stellar lithium abundances and effective temperatures. For reference, we also include the usual trend of $\mathrm{A}(\mathrm{Li})$ as a function of temperature for red giant stars from a sample of 599 giants in Brown et al. (1989), hereafter B89. Of these stars, 328 had only upper limits of $\mathrm{A}(\mathrm{Li})$. The $\mathrm{B} 89$ sample does not provide $v \sin i$ for any of its stars. The trend line indicates the average $\mathrm{A}(\mathrm{Li})$ in each temperature bin, the error bars are the dispersions in each bin $\left(\sigma_{\mathrm{A}(\mathrm{Li})}\right)$, and the hatched region shows where $\mathrm{A}(\mathrm{Li})$ is below the sensitivity limits of the survey. Because of the sensitivity limits, the trend line is likely to be even lower than plotted. To compare the lithium abundance of the rapid rotators to the slow rotators in a temperature-normalized way, we plot in histograms of $\Delta \mathrm{A}(\mathrm{Li}) /\left(\sigma_{\mathrm{A}(\mathrm{Li})}\right)$ Figure 2, where $\Delta \mathrm{A}(\mathrm{Li})$ is the distance that the measured $\mathrm{A}(\mathrm{Li})$ is from the B89 temperature trend. There is a clear distinction between these samples; rapid rotators have a larger fraction of stars enhanced in $\mathrm{A}(\mathrm{Li})$ compared to other stars in their temperature range. The right panel of Figure 2 includes stars from the literature, slow rotators from de Medeiros et al. (2000) and rapid rotators from Drake et al. (2002), in the same temperature range as our own sample and find a more prominent separation.

Rapid rotators that lie $1 \sigma$ or more from the trend line are listed in Table 1 , ordered by $v \sin i$. We also include Tyc3340-01195-1, with $\Delta \mathrm{A}(\mathrm{Li})=0.7 \sigma$, in this table because all three stars in the $\mathrm{B} 89$ sample defining the $\mathrm{A}(\mathrm{Li})$ trend in this temperature bin are lower than that of Tyc3340-01195-1, and two are upper limits only. To gain insight on the evolutionary stage of our giants, we plot them on the $T_{e f f}-\log g$ plane (TGP) in Figure 3. The left panel of Figure 3 includes five different Girardi et al. (2000) isochrones of $Z=0.12$ $([\mathrm{Fe} / \mathrm{H}] \approx-0.2 \mathrm{dex})$ with several major evolutionary points marked. The Li-enhanced rapid rotators in Table 1, solid symbols, do not seem to cluster near any particular evolutionary 


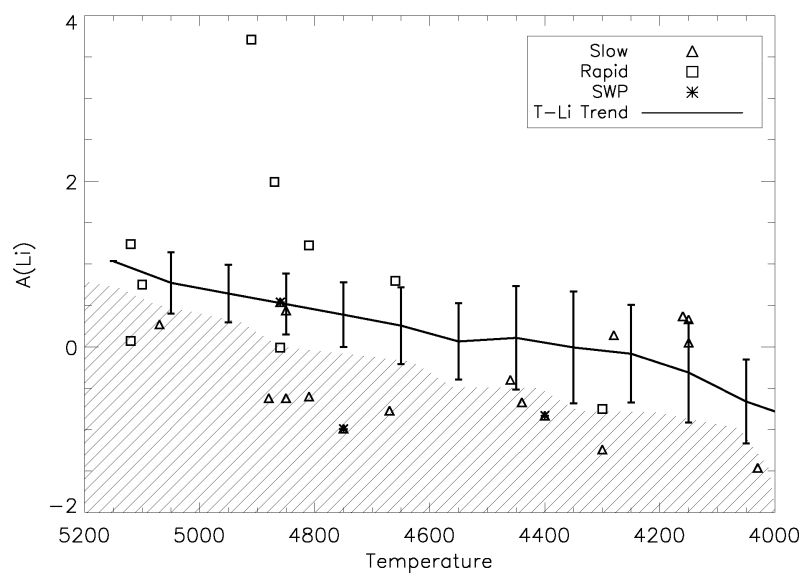

Figure 1. Lithium abundances versus effective temperature for the rapid and slow rotators. Some of the slow rotators are giant stars with planets (SWP). The line indicates the general trend of $\mathrm{A}(\mathrm{Li})$ with temperature as established from the data of $\mathrm{B} 89$. The region below the sensitivity of B89 is hatched.

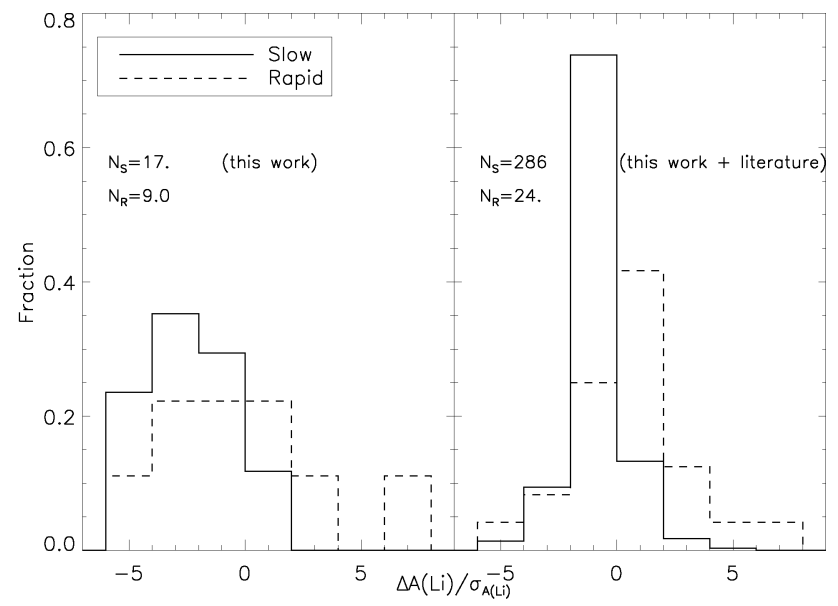

Figure 2. Histogram of the offset of $\mathrm{A}(\mathrm{Li})$ from the temperature trend $\left(\Delta \mathrm{A}(\mathrm{Li}) / \sigma_{\mathrm{A}(\mathrm{Li})}\right)$ of the rapid and slow rotator samples. Histograms on the left are from this work, while histograms on the right add stars from de Medeiros et al. (2000) and Drake et al. (2002).

stage. In the right panel of Figure 3, we show a prediction for the enhanced rotation expected from planet ingestion for the modeled future evolution of 99 known main sequence stars with planets (SWPs) by C09. The density plot shows the fraction of SWPs passing through each bin as rapid rotators compared to the number of stars passing through the bin regardless of rotation. Only SWPs expected to be rapid rotators at some point in their future RGB lifetimes are included. From this plot, we see that the Li-enhanced rapid rotators do tend to coincide with regions that have higher fractions of SWPs passing through as rapid rotators. However, our giants do not completely overlap with the future evolution of the less massive, more metal rich SWPs in this parameter space.

The mean chemical abundances of lithium in rapid and slow rotating red giants are consistent with, albeit not conclusive evidence for, the hypothesis that planet accretion is responsible for spinning up rapid rotators and that the lithium enhancement is a chemical signature of such accretion. This evidence includes (1) differences in $\mathrm{A}(\mathrm{Li})$ between the 
Table 1. Stars with unusually high $\mathrm{A}(\mathrm{Li})$ for their effective temperature, ordered by $v \sin i$.

\begin{tabular}{lcccc}
\hline Star & $\mathbf{v} \sin \mathbf{i}\left(\mathrm{km} \mathrm{s}^{-1}\right)$ & $\mathbf{A}(\mathbf{L i})(\mathbf{d e x})$ & $\mathbf{T}_{\mathbf{e f f}}(\mathbf{K})$ & {$[\mathbf{F e} / \mathbf{H}]$} \\
\hline G0928+73.2600 & 8.4 & 3.71 & 4910 & -0.25 \\
G0804+39.4755 & 9.3 & 1.23 & 4810 & -0.39 \\
Tyc3340-01195-1 & 9.3 & 1.24 & 5120 & -0.18 \\
Tyc0647-00254-1 & 10.0 & 1.99 & 4870 & 0.01 \\
Tyc5904-00513-1 & 13.0 & 0.80 & 4660 & -0.47 \\
\hline
\end{tabular}

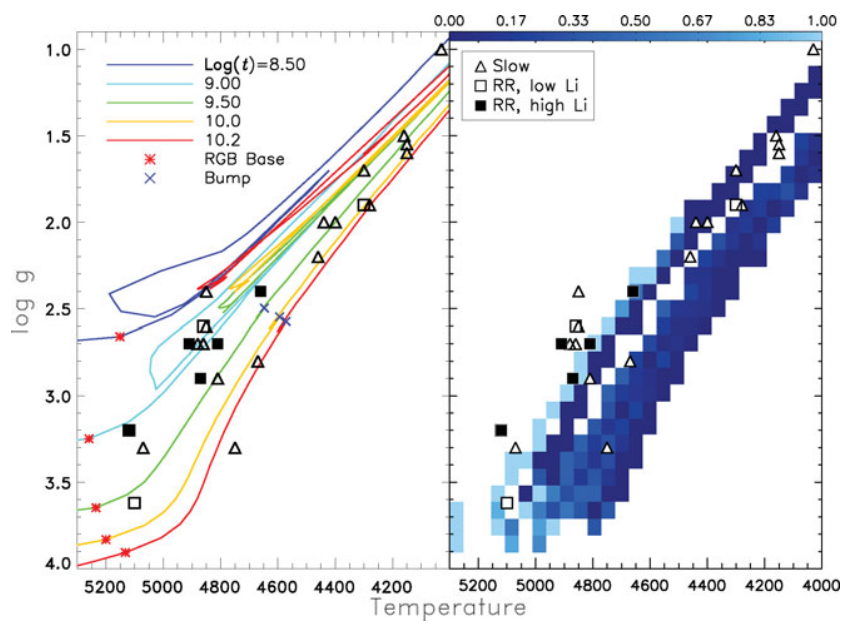

Figure 3. Temperature and $\log g$ of giants. Triangles and squares are slow and rapid rotators, respectively. (L) Solid lines give $[\mathrm{Fe} / \mathrm{H}]=0.20$ isochrones for five ages. (R) Color scale indicates the fraction of exoplanet host stars passing through each bin as rapid rotators from C09.

slow and rapid rotators, as illustrated in Figure 2, (2) no clustering of the high A(Li) rapid rotators on the TGP as would be expected if lithium was internally regenerated at a specific evolutionary stage, and (3) high $\mathrm{A}(\mathrm{Li})$ rapid rotators on the TGP coincident with where one expects to see planet accretion induced spin-up. However, much work remains. In addition to completing the lithium analysis on the remainder of our sample, we intend to look for ancillary evidence of chemical enrichment from planet accretion including enhancements of ${ }^{12} \mathrm{C}$ and refractory elements in the rapid rotators.

\section{References}

Bizyaev, D., Smith, V. V., Arenas, J., Geisler, D., Majewski, S. R., Patterson, R. J., Cunha, K., Del Pardo, C., Suntzef, N. B., \& Gieren, W. 2006, AJ, 131, 1784

Brown, J. A., Sneden, C., Lambert, D. L., \& Dutchover, E. J. 1989, ApJS, 71, 293

Carlberg, J. K., Majewski, S. R., \& Arras, P. 2009, ApJ, 700, 832

de Medeiros, J. R. \& Mayor, M. 1999, A\&AS, 139, 433

de Medeiros, J. R., do Nascimento, J. D., Jr., Sankarankutty, S., Costa, J. M., \& Maia, M. R. G. 2000, A\&A, 363, 239

Drake, N. A., de la Reza, R., da Silva, L., \& Lambert, D. L. 2002, AJ, 123, 2703

Girardi, L., Bressan, A., Bertelli, G., \& Chiosi, C. 2000, A\& $A S, 141$,

Kurucz, R. L. 1994, http://kurucz.harvard.edu

Sneden, C. A. 1973, PhD thesis, The University of Texas at Austin 